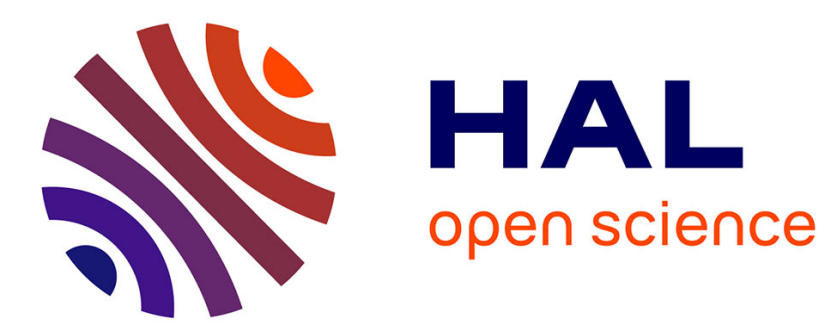

\title{
Propriété intellectuelle
}

\author{
Melanie Dulong de Rosnay
}

\section{To cite this version:}

Melanie Dulong de Rosnay. Propriété intellectuelle. Divina Frau-Meigs; Alain Kiyindou. La diversité culturelle à l'ère du numérique: glossaire critique, La Documentation Française, p. 250-253, 2014. halshs-01078706

\section{HAL Id: halshs-01078706 https://shs.hal.science/halshs-01078706}

Submitted on 9 Mar 2015

HAL is a multi-disciplinary open access archive for the deposit and dissemination of scientific research documents, whether they are published or not. The documents may come from teaching and research institutions in France or abroad, or from public or private research centers.
L'archive ouverte pluridisciplinaire HAL, est destinée au dépôt et à la diffusion de documents scientifiques de niveau recherche, publiés ou non, émanant des établissements d'enseignement et de recherche français ou étrangers, des laboratoires publics ou privés. 
Melanie Dulong de Rosnay, Propriété intellectuelle, in Divina Frau-Meigs et Alain Kiyindou (dir.), Glossaire critique sur la diversité culturelle à l'ère du numérique, Commission Nationale Française de l'UNESCO, La Documentation Française, Janvier 2015, p. 250-253.

\section{Propriété intellectuelle}

La propriété intellectuelle est la branche du droit qui régule la circulation de la création et de l'innovation dans la société. Elle comprend notamment le droit d'auteur, le droit des brevets d'invention, les marques, les expressions culturelles traditionnelles et le folklore. Cette fiction juridique vise à établir une exclusivité temporaire sur l'exploitation de l'immatériel. L'expiration de ce monopole signale l'entrée de l'oeuvre ou de l'invention dans le domaine public. Certaines créations ne remplissent pas les conditions de forme pour bénéficier d'un droit de propriété intellectuelle et sont également dans le domaine public; il s'agit des idées et connaissances générales, considérées de libre parcours et appartenant à tous.

Les titulaires de droits de propriété intellectuelle doivent délivrer une autorisation avant chaque utilisation de leurs création, qu'ils peuvent octroyer contre une rémunération. Certaines utilisations échappent à ce modèle, elles sont appelées les exceptions et limitations. Ce régime dispense de la nécessité de solliciter une autorisation préalable à chaque utilisation et de verser une rémunération, et en cela cherche à garantir un accès équitable. Les exceptions au droit d'auteur que constituent le droit de parodie ou de citation rendent possible l'expression critique sur une oeuvre préexistante sans contrôle et possible censure de la part de son auteur et donc une diversité des points de vue. Les droits exclusifs de titulaires de brevets peuvent également être limités par des licences obligatoires pour éviter de bloquer la concurrence et donc la diversité culturelle.

Le droit d'auteur s'est développé avec l'imprimerie, il est révisé à l'apparition de chaque technique de reproductibilité qui transforme les modes de reproduction et de dissémination des oeuvres : la radio et la photographie, la photocopie ou le numérique. La conjonction du numérique et des réseaux permet de reproduire et de diffuser gratuitement tout document. Cette opportunité est source de tensions entre les industries culturelles et le public. Elles sont reflétées dans le 16ème objectif du Plan d'action pour la mise en oeuvre de la Déclaration de l'UNESCO sur la diversité culturelle : "assurer la protection du droit d'auteur et des droits qui lui sont associés, dans l'intérêt du développement de la créativité contemporaine et d'une rémunération équitable du travail créatif, tout en défendant un droit public d'accès à la culture, conformément à l'article 27 de la Déclaration universelle des droits de l'homme".

En effet, le droit d'auteur peut être perçu d'un côté comme une protection des créateurs et des auxilliaires de la création que sont les interprètes, les producteurs audiovisuels et les entreprises de communication, radios et télévisions. Le droit d'auteur procure une rémunération lors de chaque utilisation des oeuvres, soutenant en principe la diversité culturelle dans la mesure où le financement de la production culturelle va apporter un revenu aux créateurs et permettre aux producteurs de soutenir les prochains créateurs. Dans la pratique, la diversité culturelle est limitée par la concentration des industries culturelles et la médiatisation de certains artistes surexposés. Le numérique change la donne avec la possibilité de se produire et se distribuer sans intermédiaire. Le phénomène de la longue traîne caractérise le développement du nombre de créations à faible diffusion, correspondant à des expressions culturelles de niche. La rareté dans un contexte d'abondance de l'offre culturelle en ligne se déplace avec le numérique vers le temps d'attention et le filtre des moteurs de recherche. Les réseaux pair-à-pair permettent la diffusion des oeuvres rares qui ne trouvent pas de canaux de diffusion commerciaux. Face à ces possibilités de reproduction et de distribution non autorisées offertes par les technologies du numérique, les entreprises du 
divertissement demandent une extension de la propriété intellectuelle et de son application pour limiter la concurrence de la distribution gratuite par les pairs. Les adaptations du droit d'auteur au numérique développent des couches de droits supplémentaires pour protéger juridiquement les mesures de protection techniques qui limitent les possibilités pratiques d'accès et de reproduction des fichiers numériques, qui deviennent moins attractifs pour le consommateur que les fichiers non bridés disponibles sur les réseaux pair à pair.

D'un autre côté, les droits de propriété intellectuelle peuvent donc limiter l'accès à la culture, la créativité et la diversité culturelle quand ils sont utilisés de manière restrictive ou extensive. L'extension de la durée des droits va empêcher la réappropriation et la réédition d'oeuvres du domaine public. Une redevance élevée va bloquer l'accès effectif à une oeuvre et réduire l'offre culturelle. Tous les créateurs ont besoin d'accéder et réutiliser des oeuvres préexistantes, mais l'évolution de la propriété intellectuelle dans le numérique conduit à réduire le champ des exceptions. Ainsi, les exceptions au droit d'auteur ne sont pas adaptées aux pratiques de remix et aux expressions culturelles individuelles. Il est difficile pour un auteur individuel de négocier des droits pour reprendre des samples ou fragment d'oeuvres sans passer par une structure intermédiaire qui risque de filtrer les goûts et in fine limiter la diversité culturelle par les choix éditoriaux et la concentration des médias. En France, le droit de citation ne s'applique qu'aux oeuvres littéraires, excluant d'inclure des extraits d'oeuvres audiovisuelles dans un mashup, et l'exception pour les utilisations dans le cadre de l'enseignement et la recherche ne favorisent pas la diversité culturelle à l'ère du numérique. En effet, les oeuvres d'art contemporain ne peuvent pas être reproduites dans les supports de cours ou les articles, et seules les oeuvres cinématographiques diffusés sur les chaînes herziennes peuvent être montrées en classe, l'exception pédagogique ne couvrant pas les films distribués sur le câble ou en ligne dans les lieux où la production indépendante est diffusée et la diversité culturelle plus large. De même, les chercheurs et les bibliothèques voient leur accès à une diversité d'expressions limité en raison de la multiplication des bouquets numériques vendus par les éditeurs de revues scientifiques. Face à cette concentration de l'offre, le mouvement pour l'accès ouvert à la recherche favorise la bibliodiversité et la dissémination de la connaissance si tous, chercheurs et non chercheurs, peuvent accéder gratuitement à des articles dans d'autres disciplines et d'autres langues que celles des portails dominés par les grands groupes anglo-saxons.

Les biens communs et les licences libres et ouvertes comme Creative Commons constituent un mode alternatif de diffusion des oeuvres couvertes par un droit de propriété intellectuelle puisque la reproduction et la diffusion sur les réseaux sont autorisés à l'avance quelque soit le type de licence. Les options de ces licences concernent l'attribution des auteurs, l'intégrité des oeuvres et l'utilisation commerciale. Cette nouvelle mise en oeuvre des droits tire partie des possibilités techniques offertes par le numérique en terme de distribution et de modification et des changements d'usages contrairement aux lois sur le droit d'auteur qui cherchent à freiner le développement des échanges sur les réseaux et des réappropriations créatives et ne considèrent la création que comme une marchandise.

L'incidence du numérique sur la diversité culturelle concerne essentiellement le droit d'auteur, les droits voisins et le droit des bases de données. Mais une autre branche de la propriété intellectuelle peut également être concernée. Il s'agit des expressions traditionnelles et du folklore. Leur réappropriation par les sociétés occidentales et les opportunités de diffusion en ligne peuvent être source de diversité culturelle comme d'appropriation exclusive ou illicite. Le risque est réel à l'ère du numérique de déplacer l'équilibre de la propriété intellectuelle vers les titulaires de droits dans les pays du Nord et de limiter l'accès à la connaissance et au patrimoine culturel immatériel et les droits du public et des pays du Sud. Les modèles des biens communs, du libre et de l'accès aux savoirs s'appuient sur les opportunités offertes par le numérique pour partager la propriété intellectuelle de manière plus équitable et collective et produire une création plus diverse. 


\section{Bibliographie}

Christoph Beat Graber, Mira Burri Nenova (ed.), Intellectual Property and Traditional Cultural Expressions in a Digital Environment, Edward Elgar Publishing, 2008.

Mélanie Dulong de Rosnay, Hervé Le Crosnier, Propriété Intellectuelle, Géopolitique et Mondialisation, CNRS Edition, coll. Essentiels d'Hermès, 2013.

Joëlle Farchy, « Promouvoir la diversité culturelle », Questions de communication, 13 | 2008, 171195.

Jacques Vetois (dir.), La propriété intellectuelle emportée par le numérique ? Terminal Technologie de l'information, culture et société, n¹02, L'Harmattan, 2009.

Michel Vivant, Propriété intellectuelle et mondialisation : la propriété intellectuelle est-elle une marchandise ?, Paris, Dalloz, coll. « Thèmes et commentaires », 2004. 